CUBO A Mathematical Journal

Vol.12, № 03 , (99-120). October 2010

\title{
Self-Dual and Anti-Self-Dual Solutions of Discrete Yang-Mills Equations on a Double Complex
}

\author{
VOLODYMYR SUSHCH \\ Koszalin University of Technology, \\ Sniadeckich 2, 75-453 Koszalin, Poland \\ email: volodymyr.sushch@tu.koszalin.pl
}

\begin{abstract}
We study a discrete model of the $S U(2)$ Yang-Mills equations on a combinatorial analog of $\mathbb{R}^{4}$. Self-dual and anti-self-dual solutions of discrete Yang-Mills equations are constructed. To obtain these solutions we use both techniques of a double complex and the quaternionic approach. Interesting analogies between instanton, anti-instanton solutions of discrete and continual self-dual, anti-self-dual equations are also discussed.
\end{abstract}

\section{RESUMEN}

Estudiamos el modelo discreto de las ecuaciones de Yang-Mills $S U(2)$ sobre un análogo combinatório de $\mathbb{R}^{4}$. Soluciones auto-dual y anti-auto-dual para las ecuaciones discretas de Yang-Mills son construidas. Para obtener estas soluciones usamos las técnicas de doble complejo y abordage cuaternionico. Interesantes analogías entre soluciones instantones y anti-instantones de ecuaciones discretas y continuas auto-dual y anti-auto-dual son discutidas.

Key words and phrases: Yang-Mills equations, self-dual and anti-self-dual equations, instantons and anti-instantons, difference equations.

Math. Subj. Class.: 81T13, 39A12. 


\section{Introduction}

It is well known that the self-dual and anti-self-dual connections are the absolute minima of the Lagrangian for a 4-dimensional non-abelian gauge theory. The first self-dual solution the one instanton - to the $S U(2)$ Yang-Mills equations on $\mathbb{R}^{4}$ was obtained by Belavin et al [3]. Later other more general multi-instanton solutions were described in $[5,11]$. Since then numerous extensions have been made. Classical references are the books by Atiyah [1], Freed and Uhlenbeck [8].

In this paper we study a discrete analog of the $S U(2)$ Yang-Mills equations on a combinatorial analog of $\mathbb{R}^{4}$. The ideas presented here are strongly influenced by book of Dezin [6]. We develop discrete models of some objects in differential geometry, including the Hodge star operator, the differential and the covariant exterior differential operator, in such a way that they preserve the geometric structure of their continual analogs. We continue the investigations which were originated in $[7,19,20,21]$. The purpose of this paper is to construct the self-dual and anti-self-dual solutions of discrete $S U(2)$ Yang-Mills equations which imitate the corresponding solutions of continual theory. The geometrical discretisation techniques used here extend those introduced in [6] and [19]. A combinatorial model of $\mathbb{R}^{4}$ based on the use of the double complex construction is taken from [21].

There are many other approaches to the discretisation of Yang-Mills theories. Numerous papers have been written on this subject. See, for example, $[2,4,9,10,12,13,15,18,16]$ and the references therein. Most of them are based on the lattice discretisation scheme. However, in the case of the lattice formulation there are difficulties in keeping geometrical properties of an origin gauge theory. An alternative geometrical discretisation scheme of a field theory can be found in [17].

The paper is organized as follows. In Section 2 we review some basic facts of the $S U(2)$ Yang-Mills theory on $\mathbb{R}^{4}$. We begin by recalling the connection between the Lie group $S U(2)$ and the space of quaternions. Finally, we write down the basic instanton and anti-instanton solutions in quaternionic form. The notations here are compiled from [1] and [14].

Section 3 contains a brief summary of definitions and properties due to the double complex construction. We repeat here the relevant material from [21]. This article is also the main reference for this section. In particular, we introduce discrete matrix-valued forms (analog of differential forms) and define analogs of the main continual operations on them.

In Section 4 using the quaternionic approach we present the discrete Yang-Mills equations. We write out components of the discrete curvature 2-form in quaternionic form. The discrete self-dual and anti-self-dual equations are described. We try to be as close to con- 
tinual $S U(2)$ Yang-Mills theory as possible. Hence we discuss conditions when the discrete curvature will be $s u(2)$-valued.

Finally, Section 5 is devoted to self-dual and anti-self-dual solutions of the discrete YangMills equations. We construct these solutions as discrete quaternionic 1-forms and discuss some analogies with continual instanton and anti-instanton solutions.

\section{Quaternions and $S U(2)$-Connection}

In this section we briefly recall some well known settings of the smooth Yang-Mills theory in Euclidean 4-dimensional space (see, for example, [14]).

We begin with a brief review of some preliminaries about quaternions. The quaternions are formed from real numbers by adjoining three symbols $\mathbf{i}, \mathbf{j}, \mathbf{k}$ and an arbitrary quaternion $x$ can be written as

$$
x=x_{1}+x_{2} \mathbf{i}+x_{3} \mathbf{j}+x_{4} \mathbf{k},
$$

where $x_{1}, x_{2}, x_{3}, x_{4} \in \mathbb{R}$. The symbols $\mathbf{i}, \mathbf{j}, \mathbf{k}$ satisfy the following identities

$$
\begin{aligned}
& \mathbf{i}^{2}=\mathbf{j}^{2}=\mathbf{k}^{2}=-1, \\
& \mathbf{i j}=-\mathbf{j i}=\mathbf{k}, \quad \mathbf{j k}=-\mathbf{k} \mathbf{j}=\mathbf{i}, \quad \mathbf{k i}=-\mathbf{i k}=\mathbf{j} .
\end{aligned}
$$

It is clear that the space of quaternions is isomorphic to $\mathbb{R}^{4}$. By analogy with the complex numbers $x_{1}$ is called the real part of $x$ and $x_{2} \mathbf{i}+x_{3} \mathbf{j}+x_{4} \mathbf{k}$ is called the imaginary part. In further we will write

$$
\operatorname{Im} x=x_{2} \mathbf{i}+x_{3} \mathbf{j}+x_{4} \mathbf{k} .
$$

The conjugate quaternion of $x$ is defined by

$$
\bar{x}=x_{1}-x_{2} \mathbf{i}-x_{3} \mathbf{j}-x_{4} \mathbf{k} .
$$

Then the norm $|x|$ of a quaternion can be introduced as follows

$$
|x|^{2}=x \bar{x}=x_{1}^{2}+x_{2}^{2}+x_{3}^{2}+x_{4}^{2} .
$$

If $x \neq 0$, then it has a unique inverse $x^{-1}$ given by

$$
x^{-1}=\bar{x} /|x|^{2} .
$$

The algebra of quaternions can be represented as a sub-algebra of the $2 \times 2$ complex matrices $M(2, \mathbb{C})$. We identify the quaternion $(2.1)$ with a matrix $f(x) \in M(2, \mathbb{C})$ by setting

$$
f(x)=\left(\begin{array}{cc}
x_{1}+x_{2} i & x_{3}+x_{4} i \\
-x_{3}+x_{4} i & x_{1}-x_{2} i
\end{array}\right) .
$$


Here $i$ is the imaginary unit.

It is well known that the unit quaternions, i.e., they have norm $|x|=1$, form a group and this group is isomorphic to $S U(2)$. The following $2 \times 2$ complex matrices

$$
\mathbf{i}=\left(\begin{array}{cc}
i & 0 \\
0 & -i
\end{array}\right), \quad \mathbf{j}=\left(\begin{array}{cc}
0 & 1 \\
-1 & 0
\end{array}\right), \quad \mathbf{k}=\left(\begin{array}{cc}
0 & i \\
i & 0
\end{array}\right)
$$

realize a representation of the Lie algebra $s u(2)$ of the group $S U(2)$. Note that multiplying by $-i$ these tree matrices we obtain the standard Pauli matrices. Matrices (2.6) correspond to the units $\mathbf{i}, \mathbf{j}, \mathbf{k}$ given by (2.2). Thus the Lie algebra $s u(2)$ can be viewed as the pure imaginary quaternions with basis $\mathbf{i}, \mathbf{j}, \mathbf{k}$.

Let now $A$ be an $S U(2)$-connection. This means that $A$ is an $s u(2)$-valued 1-form and we can write

$$
A=\sum_{\mu} A_{\mu}(x) d x^{\mu}
$$

where $A_{\mu}(x) \in s u(2)$ and $x=\left(x_{1}, \ldots, x_{4}\right)$ is a point of $\mathbb{R}^{4}$. The connection $A$ is also called a gauge potential. Define a gauge transformation by a function $g(x)$ taking value in $S U(2)$. Then the gauge potential $A$ must transform like

$$
A \rightarrow g^{-1} A g+g^{-1} d g
$$

Let us define the curvature 2 -form $F$ by

$$
F=d A+A \wedge A
$$

where $\wedge$ denotes the exterior multiplication.

Consider also the covariant exterior differential operator $d_{A}$ given by

$$
d_{A} \Omega=d \Omega+A \wedge \Omega+(-1)^{p+1} \Omega \wedge A
$$

where $\Omega$ is a $s u(2)$-valued $p$-form.

The Yang-Mills action $\mathrm{S}$ can be expressed in terms of the 2 -forms $F$ and $* F$ as

$$
S=-\operatorname{tr} \int_{\mathbb{R}^{4}} F \wedge * F
$$

where $*$ is the Hodge star operator. In $\mathbb{R}^{4}$ the operator $*^{2}$ is either an involution or antiinvolution, i.e., $*^{2}= \pm 1$. The Yang-Mills Lagrangian $L=-\operatorname{tr}(F \wedge * F)$ is invariant under the gauge transformation (2.8). By the physical requirement it is clear that the action $S$ should be finite. Hence the curvature $F$ should be square integrable. This means that $F \rightarrow 0$ as $|x| \rightarrow \infty$. 
Consequently, we must describe the boundary condition at infinity for the connection $A$. By virtue of gauge freedom (2.8) we have

$$
A \sim g^{-1} d g \quad \text { as } \quad|x| \rightarrow \infty,
$$

where $\sim$ implies asymptotic behaviour. Here and subsequently we do not specify the rate of decay.

Written in terms of the covariant exterior differential operator $d_{A}$ the Euler-Lagrange equations for the extrema of (2.11) have the form

$$
d_{A} F=0, \quad d_{A} * F=0 .
$$

These equations are the Yang-Mills equations. The first equation of (2.13) is known also as the Bianchi identity. In 4-dimensional Yang-Mills theories the following equations

$$
F=* F, \quad F=-* F
$$

are called self-dual and anti-self-dual respectively. These equations are first-order non-linear equations for the potential $A$ which imply the second-order Yang-Mills equations (2.13). Solutions of (2.14) - the self-dual and anti-self-dual connections - are called also instantons and anti-instantons [8]. It is known that the self-dual and anti-self-dual connections are the absolute minima of the action $S$.

The connection 1-form $A$ can be defined also as taking values in the space of pure imaginary quaternions. To express $A$ in quaternion form we consider the quaternion differential

$$
d x=d x_{1}+d x_{2} \mathbf{i}+d x_{3} \mathbf{j}+d x_{4} \mathbf{k}
$$

and the conjugate quaternion of $d x$

$$
d \bar{x}=d x_{1}-d x_{2} \mathbf{i}-d x_{3} \mathbf{j}-d x_{4} \mathbf{k} .
$$

Let $f(x)$ be a function of the quaternion variable $x$ with quaternion values. Then we can write $A$ as

$$
A=\operatorname{Im}(f(x) d x)
$$

where

$$
f(x)=f_{1}(x)+f_{2}(x) \mathbf{i}+f_{3}(x) \mathbf{j}+f_{4}(x) \mathbf{k} .
$$

Using the rules of multiplication (2.2) we have

$$
\begin{aligned}
& A_{1}(x)=f_{2}(x) \mathbf{i}+f_{3}(x) \mathbf{j}+f_{4}(x) \mathbf{k}, \\
& A_{2}(x)=f_{1}(x) \mathbf{i}+f_{4}(x) \mathbf{j}-f_{3}(x) \mathbf{k},
\end{aligned}
$$




$$
\begin{aligned}
& A_{3}(x)=-f_{4}(x) \mathbf{i}+f_{1}(x) \mathbf{j}+f_{2}(x) \mathbf{k}, \\
& A_{4}(x)=f_{3}(x) \mathbf{i}-f_{2}(x) \mathbf{j}+f_{1}(x) \mathbf{k} .
\end{aligned}
$$

Using (2.15) we can rewrite (2.9) as follows

$$
F=\operatorname{Im}(d f(x) \wedge d x+f(x) d x \wedge f(x) d x) .
$$

Note that calculation of the imaginary part of $f(x) d x$ and computing its curvature commute.

Let us take the following expression for $f(x)$ :

$$
f(x)=\frac{\bar{x}}{1+|x|^{2}} .
$$

Then the connection 1-form $A$ is defined by

$$
A=\operatorname{Im}\left\{\frac{\bar{x} d x}{1+|x|^{2}}\right\} .
$$

The explicit components $A_{\mu}$ can be written as

$$
\begin{array}{ll}
A_{1}(x)=\frac{-x_{2} \mathbf{i}-x_{3} \mathbf{j}-x_{4} \mathbf{k}}{1+|x|^{2}}, & A_{2}(x)=\frac{x_{1} \mathbf{i}-x_{4} \mathbf{j}+x_{3} \mathbf{k}}{1+|x|^{2}}, \\
A_{3}(x)=\frac{x_{4} \mathbf{i}+x_{1} \mathbf{j}-x_{2} \mathbf{k}}{1+|x|^{2}}, & A_{4}(x)=\frac{-x_{3} \mathbf{i}+x_{2} \mathbf{j}+x_{1} \mathbf{k}}{1+|x|^{2}} .
\end{array}
$$

Putting (2.17) in (2.16) we get the pure imaginary expression

$$
F=\frac{d \bar{x} \wedge d x}{\left(1+|x|^{2}\right)^{2}} .
$$

It is easy to show that the 2-form $d \bar{x} \wedge d x$ is anti-self-dual. Hence $F$ is anti-self-dual too and the connection (2.18) describes an anti-instanton. See for details [1].

Similarly, if we take

$$
A=\operatorname{Im}\left\{\frac{x d \bar{x}}{1+|x|^{2}}\right\},
$$

then we obtain the self-dual 2-form

$$
F=\frac{d x \wedge d \bar{x}}{\left(1+|x|^{2}\right)^{2}} .
$$

Thus the curvature is self-dual and (2.21) describes an instanton .

\section{Double Complex}

We will need the double complex construction described in [21]. In with section for the convenience of the reader we repeat the relevant material from [21] without proofs, thus making our presentation self-contained. 
Let the tensor product $C(4)=C \otimes C \otimes C \otimes C$ of an 1-dimensional complex $C$ be a combinatorial model of Euclidean space $\mathbb{R}^{4}$ (see for details also [6]). The 1-dimensional complex $C$ is defined in the following way. Let $C^{0}$ denotes the real linear space of 0 -dimensional chains generated by basis elements $x_{j}$ (points), $j \in \mathbb{Z}$. It is convenient to introduce the shift operators $\tau, \sigma$ in the set of indices by

$$
\tau j=j+1, \quad \sigma j=j-1 .
$$

We denote the open interval $\left(x_{j}, x_{\tau j}\right)$ by $e_{j}$. We'll regards the set $\left\{e_{j}\right\}$ as a set of basis elements of the real linear space $C^{1}$ of 1-dimensional chains. Then the 1-dimensional complex (combinatorial real line) is the direct sum of the introduced spaces $C=C^{0} \oplus C^{1}$. The boundary operator $\partial$ on the basis elements of $C$ is given by

$$
\partial x_{j}=0, \quad \partial e_{j}=x_{\tau j}-x_{j} .
$$

The definition is extended to arbitrary chains by linearity.

Multiplying the basis elements $x_{j}, e_{j}$ in various ways we obtain basis elements of $C(4)$. Let $s_{k}^{(p)}$, where $k=\left(k_{1}, k_{2}, k_{3}, k_{4}\right)$ and $k_{i} \in \mathbb{Z}$, be an arbitrary basis element of $C(4)$. Then a $p$-dimensional chain is given by

$$
c_{p}=\sum_{k} \sum_{p} c_{(p)}^{k} s_{k}^{(p)}, \quad c_{(p)}^{k} \in \mathbb{R} .
$$

We suppose that the superscript $(p)$ contains the whole requisite information about the quantity and places of 1-dimensional elements $e_{j}$ in $s_{k}^{(p)}$. For example, the 1-dimensional basis elements $e_{k}^{i}$ of $C(4)$ can be written as

$$
\begin{array}{ll}
e_{k}^{1}=e_{k_{1}} \otimes x_{k_{2}} \otimes x_{k_{3}} \otimes x_{k_{4}}, & e_{k}^{2}=x_{k_{1}} \otimes e_{k_{2}} \otimes x_{k_{3}} \otimes x_{k_{4}}, \\
e_{k}^{3}=x_{k_{1}} \otimes x_{k_{2}} \otimes e_{k_{3}} \otimes x_{k_{4}}, & e_{k}^{4}=x_{k_{1}} \otimes x_{k_{2}} \otimes x_{k_{3}} \otimes e_{k_{4}}
\end{array}
$$

and for the 2-dimensional basis elements $\varepsilon_{k}^{i j}$ we have

$$
\begin{array}{ll}
\varepsilon_{k}^{12}=e_{k_{1}} \otimes e_{k_{2}} \otimes x_{k_{3}} \otimes e_{k_{4}}, & \varepsilon_{k}^{23}=x_{k_{1}} \otimes e_{k_{2}} \otimes e_{k_{3}} \otimes x_{k_{4}}, \\
\varepsilon_{k}^{13}=e_{k_{1}} \otimes x_{k_{2}} \otimes e_{k_{3}} \otimes x_{k_{4}}, & \varepsilon_{k}^{24}=x_{k_{1}} \otimes e_{k_{2}} \otimes x_{k_{3}} \otimes e_{k_{4}}, \\
\varepsilon_{k}^{14}=e_{k_{1}} \otimes x_{k_{2}} \otimes x_{k_{3}} \otimes e_{k_{4}}, & \varepsilon_{k}^{34}=x_{k_{1}} \otimes x_{k_{2}} \otimes e_{k_{3}} \otimes e_{k_{4}} .
\end{array}
$$

Using (3.2) we define the boundary operator $\partial$ on chains of $C(4)$ in the following way: if $c_{p}, c_{q}$ are chains of the indicated dimension, belonging to the complexes being multiplied, then

$$
\partial\left(c_{p} \otimes c_{q}\right)=\partial c_{p} \otimes c_{q}+(-1)^{p} c_{p} \otimes \partial c_{q} .
$$

For example, for the basis element $\varepsilon_{k}^{24}$ we have

$$
\partial \varepsilon_{k}^{24}=\partial\left(x_{k_{1}} \otimes e_{k_{2}}\right) \otimes x_{k_{3}} \otimes e_{k_{4}}-x_{k_{1}} \otimes e_{k_{2}} \otimes \partial\left(x_{k_{3}} \otimes e_{k_{4}}\right)
$$




$$
\begin{aligned}
& =\partial x_{k_{1}} \otimes e_{k_{2}} \otimes x_{k_{3}} \otimes e_{k_{4}}+x_{k_{1}} \otimes \partial e_{k_{2}} \otimes x_{k_{3}} \otimes e_{k_{4}} \\
& -x_{k_{1}} \otimes e_{k_{2}} \otimes \partial x_{k_{3}} \otimes e_{k_{4}}-x_{k_{1}} \otimes e_{k_{2}} \otimes x_{k_{3}} \otimes \partial e_{k_{4}} \\
& =x_{k_{1}} \otimes x_{\tau k_{2}} \otimes x_{k_{3}} \otimes e_{k_{4}}-x_{k_{1}} \otimes x_{k_{2}} \otimes x_{k_{3}} \otimes e_{k_{4}} \\
& -x_{k_{1}} \otimes x_{k_{2}} \otimes x_{k_{3}} \otimes x_{\tau k_{4}}+x_{k_{1}} \otimes x_{k_{2}} \otimes x_{k_{3}} \otimes x_{k_{4}} .
\end{aligned}
$$

For convenience we also introduce the shift operators $\tau_{i}$ and $\sigma_{i}$ which act in the set of indices $k=\left(k_{1}, k_{2}, k_{3}, k_{4}\right), k_{i} \in \mathbb{Z}$, as

$$
\tau_{i} k=\left(k_{1}, \ldots \tau k_{i}, \ldots k_{4}\right), \quad \sigma_{i} k=\left(k_{1}, \ldots \sigma k_{i}, \ldots k_{4}\right),
$$

where $\tau$ and $\sigma$ are given by (3.1).

Let us introduce the construction of a double complex. Together with the complex $C(4)$ we consider its double, namely the complex $\tilde{C}(4)$ of exactly the same structure. Define the one-to-one correspondence

$$
*: C(4) \rightarrow \tilde{C}(4), \quad *: \tilde{C}(4) \rightarrow C(4)
$$

in the following way. Let $s_{k}^{(p)}$ be an arbitrary $p$-dimensional basis element of $C(4)$, i.e., the product $s_{k}^{(p)}=s_{k_{1}} \otimes s_{k_{2}} \otimes s_{k_{3}} \otimes s_{k_{4}}$ contains exactly $p$ of 1-dimensional elements $e_{k_{i}}$ and $4-p$ of 0 -dimensional elements $x_{k_{i}}, p=0,1,2,3,4, k_{i} \in \mathbb{Z}$. Then

$$
*: s_{k}^{(p)} \rightarrow \pm \tilde{s}_{k}^{(4-p)}, \quad *: \tilde{s}_{k}^{(4-p)} \rightarrow \pm s_{k}^{(p)},
$$

where

$$
\tilde{s}_{k}^{(4-p)}=* s_{k_{1}} \otimes * s_{k_{2}} \otimes * s_{k_{3}} \otimes * s_{k_{4}}
$$

and $* s_{k_{i}}=\tilde{e}_{k_{i}}$ if $s_{k_{i}}=x_{k_{i}}$ and $* s_{k_{i}}=\tilde{x}_{k_{i}}$ if $s_{k_{i}}=e_{k_{i}}$. In the first of mapping (3.9) we take "+" if the permutation $((p),(4-p))$ of $(1,2,3,4)$ is even and "-" if the permutation $((p),(4-p))$ is odd. Recall that in symbol $(p)$ the number of basis element is contained. For example, for the 2-dimensional basis element $\varepsilon_{k}^{13}=e_{k_{1}} \otimes x_{k_{2}} \otimes e_{k_{3}} \otimes x_{k_{4}}$ we have $* \varepsilon_{k}^{13}=-\tilde{\varepsilon}_{k}^{24}$ since the permutation $(1,3,2,4)$ is odd. The mapping $*: \tilde{s}_{k}^{(4-p)} \rightarrow \pm s_{k}^{(p)}$ is defined by analogy.

Proposition 3.1. Let $c_{r} \in C(4)$ be an r-dimensional chain (3.3). Then we have

$$
* * c_{r}=(-1)^{r(4-r)} c_{r}
$$

Proof. See [21].

Now we consider a dual object of the complex $C(4)$. Let $K(4)$ be a cochain complex with $g l(2, \mathbb{C})$-valued coefficients, where $g l(2, \mathbb{C})$ is the Lie algebra of the group $G L(2, \mathbb{C})$. Recall that $g l(2, \mathbb{C})$ consists of all complex $2 \times 2$ matrices $M(2, \mathbb{C})$ with bracket operation $[\cdot, \cdot]$. 
We suppose that the complex $K(4)$, which is a conjugate of $C(4)$, has a similar structure: $K(4)=K \otimes K \otimes K \otimes K$, where $K$ is a conjugate of the 1-dimensional complex $C$. Basis elements of $K$ can be written as $x^{j}$, $e^{j}$. Then an arbitrary basis element of $K(4)$ is given by $s_{(p)}^{k}=s^{k_{1}} \otimes s^{k_{2}} \otimes s^{k_{3}} \otimes s^{k_{4}}$, where $s^{k_{j}}$ is either $x^{k_{j}}$ or $e^{k_{j}}$. For example, we denote the 1-, 2dimensional basis elements of $K(4)$ by $e_{i}^{k}, \varepsilon_{i j}^{k}$ respectively, cf. (3.4), (3.5). For a $p$-dimensional cochain $\varphi \in K(4)$ we have

$$
\varphi=\sum_{k} \sum_{p} \varphi_{k}^{(p)} s_{(p)}^{k},
$$

where $\varphi_{k}^{(p)} \in g l(2, \mathbb{C})$. We will call cochains forms, emphasizing their relationship with the corresponding continual objects, differential forms.

We define the pairing operation $<\cdot, \cdot>$ for arbitrary basis elements $\varepsilon_{k} \in C(4), s^{k} \in K(4)$ by the rule

$$
<\varepsilon_{k}, a s^{k}>=\left\{\begin{array}{l}
0, \varepsilon_{k} \neq s_{k} \\
a, \varepsilon_{k}=s_{k}, a \in g l(2, \mathbb{C}) .
\end{array}\right.
$$

Here for simplicity the superscript ( $p$ ) is omitted. The operation (3.12) is linearly extended to cochains.

The operation $\partial$ (3.6) induces the dual operation $d^{c}$ on $K(4)$ in the following way:

$$
<\partial \varepsilon_{k}, a s^{k}>=<\varepsilon_{k}, a d^{c} s^{k}>
$$

For example, if $\varphi=\sum_{k} \varphi_{k} x^{k}$, where $x^{k}=x^{k_{1}} \otimes x^{k_{2}} \otimes x^{k_{3}} \otimes x^{k_{4}}$, is a 0 -form, then

$$
d^{c} \varphi=\sum_{k} \sum_{i=1}^{4}\left(\Delta_{i} \varphi_{k}\right) e_{i}^{k}
$$

where $\Delta_{i} \varphi_{k}=\varphi_{\tau_{i} k}-\varphi_{k}$ and $e_{i}^{k}$ is the 1-dimensional basis elements of $K(4)$. The coboundary operator $d^{c}$ is an analog of the exterior differentiation operator.

Now we describe a cochain product on the forms of $K(4)$. See [6] for details. We denote this product by $\cup$. In terms of the homology theory this is the so-called Whitney product. First we introduce the $U$-product on the chains of the 1-dimensional complex $K$. For the basis elements of $K$ the $U$-product is defined as follows

$$
x^{j} \cup x^{j}=x^{j}, \quad e^{j} \cup x^{\tau j}=e^{j}, \quad x^{j} \cup e^{j}=e^{j}, \quad j \in \mathbb{Z},
$$

supposing the product to be zero in all other case. To arbitrary forms the U-product be extended linearly. Let us introduce an $r$-dimensional complex $K(r), r=1,2,3$, in an obvious notation. Let $s_{(p)}^{k}$ be an arbitrary $p$-dimensional basis element of $K(r)$. It is convenient to write the basis element of $K(r+1)$ in the form $s_{(p)}^{k} \otimes s^{j}$, where $s_{(p)}^{k}$ is a basis element of $K(r)$ 
and $s^{j}$ is either $e^{j}$ or $x^{j}, j \in \mathbb{Z}$. Then, supposing that the $\cup$-product in $K(r)$ has been defined, we introduce it for basis elements of $K(r+1)$ by the rule

$$
\left(s_{(p)}^{k} \otimes s^{j}\right) \cup\left(s_{(q)}^{k} \otimes s^{\mu}\right)=Q(j, q)\left(s_{(p)}^{k} \cup s_{(q)}^{k}\right) \otimes\left(s^{j} \cup s^{\mu}\right),
$$

where the signum function $Q(j, q)$ is equal to -1 if the dimension of both elements $s^{j}, s_{(q)}^{k}$ is odd and to +1 otherwise. The extension of the $\cup$-product to arbitrary forms of $K(r+1)$ is linear. Note that the coefficients of forms multiply as matrices.

Proposition 3.2. Let $\varphi$ and $\psi$ be arbitrary forms of $K(4)$. Then

$$
d^{c}(\varphi \cup \psi)=d^{c} \varphi \cup \psi+(-1)^{p} \varphi \cup d^{c} \psi,
$$

where $p$ is the dimension of a form $\varphi$.

The proof of Proposition 3.2 is totally analogous to one in [6, p. 147] for the case of discrete forms with real coefficients.

The complex of the cochains $\tilde{K}(4)$ over the double complex $\tilde{C}(4)$ with the operator $d^{c}$ defined in it by (3.13) has the same structure as $K(4)$. The operation (3.8) induces the respective mapping

$$
*: K(4) \rightarrow \tilde{K}(4), \quad *: \tilde{K}(4) \rightarrow K(4)
$$

by the rule:

$$
<\tilde{c}, * \varphi>=<* \tilde{c}, \varphi>, \quad<c, * \tilde{\psi}>=<* c, \tilde{\psi}>,
$$

where $c \in C(4), \tilde{c} \in \tilde{C}(4), \varphi \in K(4), \tilde{\psi} \in \tilde{K}(4)$. Hence for the basic elements of $K(4)$ or $\tilde{K}(4)$ we have relations (3.9). It is obviously that Proposition 3.1 is true for any $r$-dimensional cochain $c^{r} \in K(4)$. So we have

$$
* * \varphi=(-1)^{r(4-r)} \varphi
$$

for any discrete $r$-form $\varphi$ on $K(4)$ and note that the same relation holds for the Hodge star operator. Thus this operator is a combinatorial analog of the Hodge star operator.

Let us introduce the following operation

$$
\tilde{\imath}: K(4) \rightarrow \tilde{K}(4), \quad \tilde{\imath}: \tilde{K}(4) \rightarrow K(4)
$$

by setting

$$
\tilde{\imath} s_{(p)}^{k}=\tilde{s}_{(p)}^{k}, \quad \tilde{l} \tilde{s}_{(p)}^{k}=s_{(p)}^{k},
$$

where $s_{(p)}^{k}$ and $\tilde{s}_{(p)}^{k}$ are basis elements of $K(4)$ and $\tilde{K}(4)$. Hence for a $p$-form $\varphi \in K(4)$ we have $\tilde{\imath} \varphi=\tilde{\varphi}$. Recall that the coefficients of $\tilde{\varphi} \in \tilde{K}(4)$ and $\varphi \in K(4)$ are the same. 
Proposition 3.3. The following hold

$$
\begin{aligned}
\tilde{\imath}^{2}=I d, \quad \tilde{\imath} * & =* \tilde{\imath}, \quad \tilde{\imath} d^{c}=d^{c} \tilde{\imath}, \\
\tilde{\imath}(\varphi \cup \psi) & =\tilde{\imath} \varphi \cup \tilde{\imath} \psi,
\end{aligned}
$$

where $\varphi, \psi \in K(4)$.

Proposition 3.4. Let $h$ be a discrete 0 -form. Then for an arbitrary $p$-form $\varphi \in K(4)$ we have

$$
\tilde{\imath} *(h \cup \varphi)=h \cup \tilde{\imath} * \varphi .
$$

Proof. See [21].

Note that the definition of inner product in the double complex and a discrete analog of the Yang-Mills actions (2.11) can be found in [21].

\section{Quaternions and Discrete Forms}

Let us consider a discrete 0 -form with coefficients belonging to $M(2, \mathbb{C})$. We put

$$
f=\sum_{k} f_{k} x^{k}
$$

where $x^{k}=x^{k_{1}} \otimes x^{k_{2}} \otimes x^{k_{3}} \otimes x^{k_{4}}$ is the 0 -dimensional basis element of $K(4), k=\left(k_{1}, k_{2}, k_{3}, k_{4}\right), k_{i} \in$ $\mathbb{Z}$. Suppose that the matrices $f_{k} \in M(2, \mathbb{C})$ look like (2.5), i. e.

$$
f_{k}=\left(\begin{array}{cc}
f_{k}^{1}+f_{k}^{2} i & f_{k}^{3}+f_{k}^{4} i \\
-f_{k}^{3}+f_{k}^{4} i & f_{k}^{1}-f_{k}^{2} i
\end{array}\right),
$$

where $f_{k}^{s} \in \mathbb{R}, s=1,2,3,4$. Then $f_{k}$ in quaternionic form can be expressed as

$$
f_{k}=f_{k}^{1}+f_{k}^{2} \mathbf{i}+f_{k}^{3} \mathbf{j}+f_{k}^{4} \mathbf{k} .
$$

Hence the form (4.1) can be considered as a discrete form with quaternionic coefficients. We will call it simply the quaternionic form when no confusion can arise. In a proper way we define the quaternionic 0 -form $\bar{f}$ with coefficients $\bar{f}_{k}$ regarded as the conjugate quaternions of $f_{k}$. Let $f^{-1}$ be the quaternionic form, where $f_{k}^{-1}$ is given by (2.4). Then we have

$$
f \cup f^{-1}=\sum_{k} f_{k} f_{k}^{-1} x^{k}=\sum_{k} x^{k} .
$$

Proposition 4.1. Let $f$ be a discrete 0 -form and $f \neq 0$. Then we have

$$
d^{c} f \cup f^{-1}=-f \cup d^{c} f^{-1} .
$$


Proof. By definition (3.14) and according to (4.4), we have $d^{c}\left(f \cup f^{-1}\right)=0$. Using Proposition 3.2 we immediately obtain (4.5).

Let us denote by $e$ the following quaternionic 1-form

$$
e=\sum_{k} e^{k}=\sum_{k}\left(e_{1}^{k}+e_{2}^{k} \mathbf{i}+e_{3}^{k} \mathbf{j}+e_{4}^{k} \mathbf{k}\right)
$$

where $e_{i}^{k}$ is the 1 -dimensional basis elements of $K(4)$. Let $A \in K(4)$ be a discrete 1 -form. We define the discrete $S U(2)$-connection $A$ to be

$$
A=\sum_{k} \sum_{i=1}^{4} A_{k}^{i} e_{i}^{k},
$$

where $A_{k}^{i} \in s u(2)$ and $k=\left(k_{1}, k_{2}, k_{3}, k_{4}\right), k_{i} \in \mathbb{Z}$. Using (4.3) and (4.6) we write (4.7) in quaternionic form as

$$
A=\operatorname{Im}(f \cup e)=\operatorname{Im}\left(\sum_{k} f_{k} e^{k}\right)
$$

Then the $A_{k}^{i}$ are given by

$$
\begin{array}{ll}
A_{k}^{1}=f_{k}^{2} \mathbf{i}+f_{k}^{3} \mathbf{j}+f_{k}^{4} \mathbf{k}, & A_{k}^{2}=f_{k}^{1} \mathbf{i}+f_{k}^{4} \mathbf{j}-f_{k}^{3} \mathbf{k}, \\
A_{k}^{3}=-f_{k}^{4} \mathbf{i}+f_{k}^{1} \mathbf{j}+f_{k}^{2} \mathbf{k}, & A_{k}^{4}=f_{k}^{3} \mathbf{i}-f_{k}^{2} \mathbf{j}+f_{k}^{1} \mathbf{k} .
\end{array}
$$

Define the quaternionic 0 -form $x$ by

$$
x=\sum_{k} \kappa x^{k}, \quad \kappa=k_{1}+k_{2} \mathbf{i}+k_{3} \mathbf{j}+k_{4} \mathbf{k},
$$

where $k_{i} \in \mathbb{Z}$. It is easy to check that

$$
d^{c} x=e
$$

Therefore we can rewrite (4.8) as

$$
A=\operatorname{Im}\left(f \cup d^{c} x\right) .
$$

Let $g$ be a quaternionic 0 -form (4.1) with the components of unit norm, i.e., $\left|g_{k}\right|=1$ for any $k$. It means that the corresponding discrete form is $S U(2)$-valued. We now define a gauge transformation for the discrete potential $A$ which is analogous to (2.8). This is

$$
A \rightarrow g^{-1} \cup A \cup g+g^{-1} \cup d^{c} g
$$

where $A$ is given by (4.8) or (4.12). Note that the gauge transformed discrete form $A$ is $s u(2)$-valued too. It is not so obviously as in the continual case but follows immediately from the definition of $\cup$-multiplication and formula (3.16). More generally, if we assume that the gauge transformation $g$ is an arbitrary quaternionic 0 -form, then we take the imaginary part 
of $g^{-1} \cup A \cup g+g^{-1} \cup d^{c} g$ in (4.13). For a deeper discussion of gauge invariant discrete models of the Yang-Mills theory we refer the reader to [19, 21].

An arbitrary discrete 2-form $F \in K(4)$ can be written as follows

$$
F=\sum_{k} \sum_{i<j} F_{k}^{i j} \varepsilon_{i j}^{k}
$$

where $F_{k}^{i j} \in g l(2, \mathbb{C}), \varepsilon_{i j}^{k}$ is the 2-dimensional basis element of $K(4)$ and $1 \leq i, j \leq 4$, $k=\left(k_{1}, k_{2}, k_{3}, k_{4}\right), k_{i} \in \mathbb{Z}$. Let $F$ is given by

$$
F=d^{c} A+A \cup A .
$$

Combining (4.7) and (4.15) and using (3.12), (3.13) and (3.15), we obtain

$$
F_{k}^{i j}=\Delta_{i} A_{k}^{j}-\Delta_{j} A_{k}^{i}+A_{k}^{i} A_{\tau_{i} k}^{j}-A_{k}^{j} A_{\tau_{j} k}^{i},
$$

where $\Delta_{i} A_{k}^{j}=A_{\tau_{i} k}^{j}-A_{k}^{j}$ and $\tau_{i} k$ is given by (3.7).

Let us define a discrete analog of the exterior covariant differentiation operator (2.10) as follows

$$
d_{A}^{c} \Omega=d^{c} \Omega+A \cup \Omega+(-1)^{p+1} \Omega \cup A,
$$

where $\Omega$ is an arbitrary $p$-form of $K(4)$ looking like (3.11). Then a discrete analog of Equations (2.13) can be written as

$$
d_{A}^{c} F=0, \quad d_{A}^{c} * \tilde{l} F=0,
$$

where $\tilde{\imath}$ is given by (3.18). It is easy to check that the combinatorial Bianchi identity:

$$
d^{c} F+A \cup F-F \cup A=0
$$

holds for the discrete curvature form (4.15) (cf. (2.13)).

Remark 4.2. In the continual case the curvature form $F$ (2.9) takes values in the algebra su(2) for any su(2)-valued connection form A. Unfortunately, this is not true in the discrete case because, generally speaking, the components $A_{k}^{i} A_{\tau_{i} k}^{j}-A_{k}^{j} A_{\tau_{j} k}^{i}$ of the form $A \cup A$ (see (4.16)) do not belong to su(2).

To define an $s u(2)$-valued discrete analog of the curvature 2-form we use the quaternionic form of $A$ (4.8) and put in (4.15). Then the discrete curvature form $F$ is given by

$$
F=\operatorname{Im}\left\{d^{c} f \cup e+(f \cup e) \cup(f \cup e)\right\} .
$$

It should be noted that in the discrete case calculation of the imaginary part of $f \cup e$ and computing its curvature do not commute. 
Proposition 4.3. If $A=\operatorname{Im}\left(x^{-1} \cup d^{c} x\right)$, where $x$ is given by (4.10), then $F=0$.

Proof. Using (4.5) and putting $f=x^{-1}$ in (4.20) we get

$$
\begin{aligned}
F & =\operatorname{Im}\left(d^{c}\left(x^{-1} \cup d^{c} x\right)+\left(x^{-1} \cup d^{c} x\right) \cup\left(x^{-1} \cup d^{c} x\right)\right. \\
& =\operatorname{Im}\left(d^{c} x^{-1} \cup d^{c} x-d^{c} x^{-1} \cup x \cup x^{-1} \cup d^{c} x\right) .
\end{aligned}
$$

According to (4.4) the form $x \cup x^{-1}$ has unit components. Hence

$$
d^{c} x^{-1} \cup x \cup x^{-1} \cup d^{c} x=d^{c} x^{-1} \cup d^{c} x .
$$

We now write down the components of (4.14) using quaternions. Putting (4.9) in (4.16) we find that

$$
\begin{aligned}
& F_{k}^{12}=\left(\Delta_{1} f_{k}^{1}-\Delta_{2} f_{k}^{2}-f_{k}^{3} f_{\tau_{1} k}^{3}-f_{k}^{4} f_{\tau_{1} k}^{4}-f_{k}^{3} f_{\tau_{2} k}^{3}-f_{k}^{4} f_{\tau_{2} k}^{4}\right) \mathbf{i} \\
& +\left(\Delta_{1} f_{k}^{4}-\Delta_{2} f_{k}^{3}+f_{k}^{2} f_{\tau_{1} k}^{3}+f_{k}^{4} f_{\tau_{1} k}^{1}+f_{k}^{1} f_{\tau_{2} k}^{4}+f_{k}^{3} f_{\tau_{2} k}^{2}\right) \mathbf{j} \\
& +\left(-\Delta_{1} f_{k}^{3}-\Delta_{2} f_{k}^{4}+f_{k}^{2} f_{\tau_{1} k}^{4}-f_{k}^{3} f_{\tau_{1} k}^{1}-f_{k}^{1} f_{\tau_{2} k}^{3}+f_{k}^{4} f_{\tau_{2} k}^{2}\right) \mathbf{k} \\
& -f_{k}^{2} f_{\tau_{1} k}^{1}-f_{k}^{3} f_{\tau_{1} k}^{4}+f_{k}^{4} f_{\tau_{1} k}^{3}+f_{k}^{1} f_{\tau_{2} k}^{2}+f_{k}^{4} f_{\tau_{2} k}^{3}-f_{k}^{3} f_{\tau_{2} k}^{4}, \\
& F_{k}^{13}=\left(-\Delta_{1} f_{k}^{4}-\Delta_{3} f_{k}^{2}+f_{k}^{3} f_{\tau_{1} k}^{2}-f_{k}^{4} f_{\tau_{1} k}^{1}-f_{k}^{1} f_{\tau_{3} k}^{4}+f_{k}^{2} f_{\tau_{3} k}^{3}\right) \mathbf{i} \\
& +\left(\Delta_{1} f_{k}^{1}-\Delta_{3} f_{k}^{3}-f_{k}^{2} f_{\tau_{1} k}^{2}-f_{k}^{4} f_{\tau_{1} k}^{4}-f_{k}^{4} f_{\tau_{3} k}^{4}-f_{k}^{2} f_{\tau_{3} k}^{2}\right) \mathbf{j} \\
& +\left(\Delta_{1} f_{k}^{2}-\Delta_{3} f_{k}^{4}+f_{k}^{2} f_{\tau_{1} k}^{1}+f_{k}^{3} f_{\tau_{1} k}^{4}+f_{k}^{4} f_{\tau_{3} k}^{3}+f_{k}^{1} f_{\tau_{3} k}^{2}\right) \mathbf{k} \\
& +f_{k}^{2} f_{\tau_{1} k}^{4}-f_{k}^{3} f_{\tau_{1} k}^{1}-f_{k}^{4} f_{\tau_{1} k}^{2}-f_{k}^{4} f_{\tau_{3} k}^{2}+f_{k}^{1} f_{\tau_{3} k}^{3}+f_{k}^{2} f_{\tau_{3} k}^{4}, \\
& F_{k}^{14}=\left(\Delta_{1} f_{k}^{3}-\Delta_{4} f_{k}^{2}+f_{k}^{3} f_{\tau_{1} k}^{1}+f_{k}^{4} f_{\tau_{1} k}^{2}+f_{k}^{2} f_{\tau_{4} k}^{4}+f_{k}^{1} f_{\tau_{4} k}^{3}\right) \mathbf{i} \\
& +\left(-\Delta_{1} f_{k}^{2}-\Delta_{4} f_{k}^{3}-f_{k}^{2} f_{\tau_{1} k}^{1}+f_{k}^{4} f_{\tau_{1} k}^{3}+f_{k}^{3} f_{\tau_{4} k}^{4}-f_{k}^{1} f_{\tau_{4} k}^{2}\right) \mathbf{j} \\
& +\left(\Delta_{1} f_{k}^{1}-\Delta_{4} f_{k}^{4}-f_{k}^{2} f_{\tau_{1} k}^{2}-f_{k}^{3} f_{\tau_{1} k}^{3}-f_{k}^{3} f_{\tau_{4} k}^{3}-f_{k}^{2} f_{\tau_{4} k}^{2}\right) \mathbf{k} \\
& -f_{k}^{2} f_{\tau_{1} k}^{3}+f_{k}^{3} f_{\tau_{1} k}^{2}-f_{k}^{4} f_{\tau_{1} k}^{1}+f_{k}^{3} f_{\tau_{4} k}^{2}-f_{k}^{2} f_{\tau_{4} k}^{3}+f_{k}^{1} f_{\tau_{4} k}^{4}, \\
& F_{k}^{23}=\left(-\Delta_{2} f_{k}^{4}-\Delta_{3} f_{k}^{1}+f_{k}^{4} f_{\tau_{2} k}^{2}+f_{k}^{3} f_{\tau_{2} k}^{1}+f_{k}^{1} f_{\tau_{3} k}^{3}+f_{k}^{2} f_{\tau_{3} k}^{4}\right) \mathbf{i} \\
& +\left(\Delta_{2} f_{k}^{1}-\Delta_{3} f_{k}^{4}-f_{k}^{1} f_{\tau_{2} k}^{2}+f_{k}^{3} f_{\tau_{2} k}^{4}+f_{k}^{4} f_{\tau_{3} k}^{3}-f_{k}^{2} f_{\tau_{3} k}^{1}\right) \mathbf{j} \\
& +\left(\Delta_{2} f_{k}^{2}+\Delta_{3} f_{k}^{3}+f_{k}^{1} f_{\tau_{2} k}^{1}+f_{k}^{4} f_{\tau_{2} k}^{4}+f_{k}^{4} f_{\tau_{3} k}^{4}+f_{k}^{1} f_{\tau_{3} k}^{1}\right) \mathbf{k} \\
& +f_{k}^{1} f_{\tau_{2} k}^{4}-f_{k}^{4} f_{\tau_{2} k}^{1}+f_{k}^{3} f_{\tau_{2} k}^{2}-f_{k}^{4} f_{\tau_{3} k}^{1}+f_{k}^{1} f_{\tau_{3} k}^{4}-f_{k}^{2} f_{\tau_{3} k}^{3}, \\
& F_{k}^{24}=\left(\Delta_{2} f_{k}^{3}-\Delta_{4} f_{k}^{1}+f_{k}^{4} f_{\tau_{2} k}^{1}-f_{k}^{3} f_{\tau_{2} k}^{2}-f_{k}^{2} f_{\tau_{4} k}^{3}+f_{k}^{1} f_{\tau_{4} k}^{4}\right) \mathbf{i}
\end{aligned}
$$




$$
\begin{aligned}
& +\left(-\Delta_{2} f_{k}^{2}-\Delta_{4} f_{k}^{4}-f_{k}^{1} f_{\tau_{2} k}^{1}-f_{k}^{3} f_{\tau_{2} k}^{3}-f_{k}^{3} f_{\tau_{4} k}^{3}-f_{k}^{1} f_{\tau_{4} k}^{1}\right) \mathbf{j} \\
& +\left(\Delta_{2} f_{k}^{1}+\Delta_{4} f_{k}^{3}-f_{k}^{1} f_{\tau_{2} k}^{2}-f_{k}^{4} f_{\tau_{2} k}^{3}-f_{k}^{3} f_{\tau_{4} k}^{4}-f_{k}^{2} f_{\tau_{4} k}^{1}\right) \mathbf{k} \\
& -f_{k}^{1} f_{\tau_{2} k}^{3}+f_{k}^{4} f_{\tau_{2} k}^{2}+f_{k}^{3} f_{\tau_{2} k}^{1}+f_{k}^{3} f_{\tau_{4} k}^{1}-f_{k}^{2} f_{\tau_{4} k}^{4}-f_{k}^{1} f_{\tau_{4} k}^{3}, \\
F_{k}^{34} & =\left(\Delta_{3} f_{k}^{3}+\Delta_{4} f_{k}^{4}+f_{k}^{1} f_{\tau_{3} k}^{1}+f_{k}^{2} f_{\tau_{3} k}^{2}+f_{k}^{2} f_{\tau_{4} k}^{2}+f_{k}^{1} f_{\tau_{4} k}^{1}\right) \mathbf{i} \\
& +\left(-\Delta_{3} f_{k}^{2}-\Delta_{4} f_{k}^{1}+f_{k}^{4} f_{\tau_{3} k}^{1}+f_{k}^{2} f_{\tau_{3} k}^{3}+f_{k}^{3} f_{\tau_{4} k}^{2}+f_{k}^{1} f_{\tau_{4} k}^{4}\right) \mathbf{j} \\
& +\left(\Delta_{3} f_{k}^{1}-\Delta_{4} f_{k}^{2}+f_{k}^{4} f_{\tau_{3} k}^{2}-f_{k}^{1} f_{\tau_{3} k}^{3}-f_{k}^{3} f_{\tau_{4} k}^{1}+f_{k}^{2} f_{\tau_{4} k}^{4}\right) \mathbf{k} \\
& +f_{k}^{4} f_{\tau_{3} k}^{3}+f_{k}^{1} f_{\tau_{3} k}^{2}-f_{k}^{2} f_{\tau_{3} k}^{1}-f_{k}^{3} f_{\tau_{4} k}^{4}-f_{k}^{2} f_{\tau_{4} k}^{1}+f_{k}^{1} f_{\tau_{4} k}^{2} .
\end{aligned}
$$

To obtain the components of (4.20) we must take the imaginary part of these equations.

Proposition 4.4. The discrete curvature 2-form $F$ (4.15) is su(2)-valued if and only if

$$
\begin{array}{r}
-f_{k}^{2} f_{\tau_{1} k}^{1}-f_{k}^{3} f_{\tau_{1} k}^{4}+f_{k}^{4} f_{\tau_{1} k}^{3}+f_{k}^{1} f_{\tau_{2} k}^{2}+f_{k}^{4} f_{\tau_{2} k}^{3}-f_{k}^{3} f_{\tau_{2} k}^{4}=0, \\
f_{k}^{2} f_{\tau_{1} k}^{4}-f_{k}^{3} f_{\tau_{1} k}^{1}-f_{k}^{4} f_{\tau_{1} k}^{2}-f_{k}^{4} f_{\tau_{3} k}^{2}+f_{k}^{1} f_{\tau_{3} k}^{3}+f_{k}^{2} f_{\tau_{3} k}^{4}=0, \\
-f_{k}^{2} f_{\tau_{1} k}^{3}+f_{k}^{3} f_{\tau_{1} k}^{2}-f_{k}^{4} f_{\tau_{1} k}^{1}+f_{k}^{3} f_{\tau_{4} k}^{2}-f_{k}^{2} f_{\tau_{4} k}^{3}+f_{k}^{1} f_{\tau_{4} k}^{4}=0, \\
f_{k}^{1} f_{\tau_{2} k}^{4}-f_{k}^{4} f_{\tau_{2} k}^{1}+f_{k}^{3} f_{\tau_{2} k}^{2}-f_{k}^{4} f_{\tau_{3} k}^{1}+f_{k}^{1} f_{\tau_{3} k}^{4}-f_{k}^{2} f_{\tau_{3} k}^{3}=0, \\
-f_{k}^{1} f_{\tau_{2 k}}^{3}+f_{k}^{4} f_{\tau_{2} k}^{2}+f_{k}^{3} f_{\tau_{2} k}^{1}+f_{k}^{3} f_{\tau_{4} k}^{1}-f_{k}^{2} f_{\tau_{4} k}^{4}-f_{k}^{1} f_{\tau_{4} k}^{3}=0, \\
f_{k}^{4} f_{\tau_{3} k}^{3}+f_{k}^{1} f_{\tau_{3} k}^{2}-f_{k}^{2} f_{\tau_{3} k}^{1}-f_{k}^{3} f_{\tau_{4} k}^{4}-f_{k}^{2} f_{\tau_{4} k}^{1}+f_{k}^{1} f_{\tau_{4} k}^{2}=0 .
\end{array}
$$

Proof. From the above it follows immediately.

Proposition 4.5. Let e is given by (4.6). Then the 2-form $e \cup \bar{e}$ is self-dual, i.e.,

$$
e \cup \bar{e}=* \tilde{l}(e \cup \bar{e}),
$$

and $\bar{e} \cup$ e is anti-self-dual, i.e.,

$$
\bar{e} \cup e=-* \tilde{l}(\bar{e} \cup e) .
$$

Proof. Denote

$$
e_{i}=\sum_{k} e_{i}^{k}, \quad \varepsilon_{i j}=\sum_{k} \varepsilon_{i j}^{k} .
$$

Recall that $e_{i}^{k}$ and $\varepsilon_{i j}^{k}$ are the 1-dimensional and 2-dimensional basic elements of $K(4)$ (see also (3.4) and (3.5)). From this by (3.15) we obtain $e_{i} \cup e_{j}=\varepsilon_{i j}$ and $e_{j} \cup e_{i}=-\varepsilon_{i j}$ for all $i<j$. Then we have

$$
\begin{aligned}
e \cup \bar{e} & =\left(e_{1}+e_{2} \mathbf{i}+e_{3} \mathbf{j}+e_{4} \mathbf{k}\right) \cup\left(e_{1}-e_{2} \mathbf{i}-e_{3} \mathbf{j}-e_{4} \mathbf{k}\right) \\
& =-2\left\{\left(e_{1} \cup e_{2}+e_{3} \cup e_{4}\right) \mathbf{i}+\left(e_{1} \cup e_{3}-e_{2} \cup e_{4}\right) \mathbf{j}+\left(e_{1} \cup e_{4}+e_{2} \cup e_{3}\right) \mathbf{k}\right\} \\
& =-2\left\{\left(\varepsilon_{12}+\varepsilon_{34}\right) \mathbf{i}+\left(\varepsilon_{13}-\varepsilon_{24}\right) \mathbf{j}+\left(\varepsilon_{14}+\varepsilon_{23}\right) \mathbf{k}\right\} .
\end{aligned}
$$


Using (3.17) and (3.19) we get

$$
* \tilde{l}(e \cup \bar{e})=-2 \tilde{\imath}\left\{\left(\tilde{\varepsilon}_{34}+\tilde{\varepsilon}_{12}\right) \mathbf{i}+\left(-\tilde{\varepsilon}_{24}+\tilde{\varepsilon}_{13}\right) \mathbf{j}+\left(\tilde{\varepsilon}_{23}+\tilde{\varepsilon}_{14}\right) \mathbf{k}\right\}=e \cup \bar{e} .
$$

In the same way we obtain (4.22).

Corollary 4.6. For any quaternionic 0 -form $f$ the form $f \cup e \cup \bar{e}$ is self-dual and $f \cup \bar{e} \cup e$ is anti-self-dual.

Proof. This follows immediately from (3.20).

Discrete self-dual and anti-self-dual equations (discrete analogs of Equations (2.13)) are defined by

$$
F=\tilde{\imath} * F, \quad F=-\tilde{\imath} * F,
$$

where $F$ is the discrete curvature form (4.4). Using (4.5), by the definitions of $\tilde{\imath}$ and $*$, the first equation (self-dual) of (4.23) can be rewritten as follows

$$
F_{k}^{12}=F_{k}^{34}, \quad F_{k}^{13}=-F_{k}^{24}, \quad F_{k}^{14}=F_{k}^{23} .
$$

By analogue with the continual case solutions of (4.23) (or (4.24)) are called instantons and anti-instantons respectively.

\section{Discrete Instanton and Anti-Instanton}

In further analogy with the continual case consider the discrete $S U(2)$-connection $A$. Let $A$ be the quaternionic 1-form (4.8), where the components of $f$ are given by

$$
f_{k}=\frac{\bar{\kappa}}{1+|\kappa|^{2}},
$$

where $\kappa=k_{1}+k_{2} \mathbf{i}+k_{3} \mathbf{j}+k_{4} \mathbf{k}, k_{i} \in \mathbb{Z}$. Putting the last in (4.9) we obtain

$$
\begin{array}{rlrl}
A_{k}^{1} & =\frac{-k_{2} \mathbf{i}-k_{3} \mathbf{j}-k_{4} \mathbf{k}}{1+|\kappa|^{2}}, & A_{k}^{2}=\frac{k_{1} \mathbf{i}-k_{4} \mathbf{j}+k_{3} \mathbf{k}}{1+|\kappa|^{2}}, \\
A_{k}^{3}=\frac{k_{4} \mathbf{i}+k_{1} \mathbf{j}-k_{2} \mathbf{k}}{1+|\kappa|^{2}}, & A_{k}^{4}=\frac{-k_{3} \mathbf{i}+k_{2} \mathbf{j}+k_{1} \mathbf{k}}{1+|\kappa|^{2}} .
\end{array}
$$

It is convenient to denote

$$
M_{i}=\frac{1}{\left(1+|\kappa|^{2}\right)\left(1+\left|\tau_{i} \kappa\right|^{2}\right)}, \quad i=1,2,3,4 .
$$

Recall that the shift operator $\tau_{i}$ is given by (3.7). Substituting (5.2) in (4.16) and using (5.3) we find that

$$
F_{k}^{12}=\left\{M_{1}\left(1+k_{2}^{2}-k_{1}^{2}-k_{1}\right)+M_{2}\left(1+k_{1}^{2}-k_{2}^{2}-k_{2}\right)\right\} \mathbf{i}
$$




$$
\begin{aligned}
& +\left\{M_{1}\left(k_{4} k_{1}+k_{2} k_{3}\right)-M_{2}\left(k_{3} k_{2}+k_{4} k_{1}\right)\right\} \mathbf{j} \\
& +\left\{M_{1}\left(k_{2} k_{4}-k_{1} k_{3}\right)+M_{2}\left(k_{1} k_{3}-k_{2} k_{4}\right)\right\} \mathbf{k} \\
& +M_{1}\left(k_{1} k_{2}+k_{2}\right)-M_{2}\left(k_{1} k_{2}+k_{1}\right), \\
F_{k}^{13} & =\left\{M_{1}\left(k_{2} k_{3}-k_{1} k_{4}\right)+M_{3}\left(k_{1} k_{4}-k_{2} k_{3}\right)\right\} \mathbf{i} \\
& +\left\{M_{1}\left(1+k_{3}^{2}-k_{1}^{2}-k_{1}\right)+M_{3}\left(1+k_{1}^{2}-k_{3}^{2}-k_{3}\right)\right\} \mathbf{j} \\
& +\left\{M_{1}\left(k_{1} k_{2}+k_{3} k_{4}\right)-M_{3}\left(k_{3} k_{4}+k_{1} k_{2}\right)\right\} \mathbf{k} \\
& +M_{1}\left(k_{1} k_{3}+k_{3}\right)-M_{3}\left(k_{1} k_{3}+k_{1}\right), \\
F_{k}^{14} & =\left\{M_{1}\left(k_{1} k_{3}+k_{2} k_{4}\right)-M_{4}\left(k_{2} k_{4}+k_{1} k_{3}\right)\right\} \mathbf{i} \\
& +\left\{M_{1}\left(k_{3} k_{4}-k_{1} k_{2}\right)+M_{4}\left(k_{1} k_{2}-k_{3} k_{4}\right)\right\} \mathbf{j} \\
& +\left\{M_{1}\left(1+k_{4}^{2}-k_{1}^{2}-k_{1}\right)+M_{4}\left(1+k_{1}^{2}-k_{4}^{2}-k_{4}\right)\right\} \mathbf{k} \\
& +M_{1}\left(k_{1} k_{4}+k_{4}\right)-M_{4}\left(k_{1} k_{4}+k_{1}\right), \\
F_{k}^{23} & =\left\{-M_{2}\left(k_{2} k_{4}+k_{1} k_{3}\right)+M_{3}\left(k_{1} k_{3}+k_{2} k_{4}\right)\right\} \mathbf{i} \\
& +\left\{M_{2}\left(k_{3} k_{4}-k_{1} k_{2}\right)+M_{3}\left(k_{1} k_{2}-k_{3} k_{4}\right)\right\} \mathbf{j} \\
& -\left\{M_{2}\left(1+k_{3}^{2}-k_{2}^{2}-k_{2}\right)+M_{3}\left(1+k_{2}^{2}-k_{3}^{2}-k_{3}\right)\right\} \mathbf{k} \\
& +M_{2}\left(k_{2} k_{3}+k_{3}\right)-M_{3}\left(k_{2} k_{3}+k_{2}\right), \\
F_{k}^{24} & =\left\{M_{2}\left(k_{2} k_{3}-k_{4} k_{1}\right)+M_{4}\left(k_{1} k_{4}-k_{2} k_{3}\right)\right\} \mathbf{i} \\
& +\left\{M_{2}\left(1+k_{4}^{2}-k_{2}^{2}-k_{2}\right)+M_{4}\left(1+k_{2}^{2}-k_{4}^{2}-k_{4}\right)\right\} \mathbf{j} \\
& -\left\{M_{2}\left(k_{1} k_{2}+k_{3} k_{4}\right)-M_{4}\left(k_{3} k_{4}+k_{1} k_{2}\right)\right\} \mathbf{k} \\
& +M_{2}\left(k_{2} k_{4}+k_{4}\right)-M_{4}\left(k_{2} k_{4}+k_{2}\right), \\
+ & -\left\{M_{3}\left(1+k_{4}^{2}-k_{3}^{2}-k_{3}\right)+M_{4}\left(1+k_{3}^{2}-k_{4}^{2}-k_{1} k_{4}\right)+M_{4}\left(k_{1} k_{4}+k_{2} k_{3}\right)\right\} \mathbf{j} \\
+ & M_{3}\left(k_{3} k_{4}+k_{4}\right)-M_{4}\left(k_{3} k_{4}+k_{3}\right) . \\
&
\end{aligned}
$$

Proposition 5.1. The 2-form $F$ with components $F_{k}^{i j}$ above is su(2)-valued if and only if

$$
k_{1}=k_{2}=k_{3}=k_{4} \text {. }
$$

Proof. From Proposition $4.4 F$ is $s u(2)$-valued if and only if

$$
M_{i}\left(k_{i} k_{j}+k_{j}\right)-M_{j}\left(k_{i} k_{j}+k_{i}\right)=0
$$

for any $k_{i} \in \mathbb{Z}, i, j=1,2,3,4$ and $i<j$. It follows immediately (5.4). 
Thus, the $s u(2)$-valued discrete curvature 2 -form $F$ can be written in the quaternionic form as follows

$$
F=\sum_{k, k_{i}=\mu} M_{\mu}(2-2 \mu)\left\{\left(\varepsilon_{12}^{k}-\varepsilon_{34}^{k}\right) \mathbf{i}+\left(\varepsilon_{13}^{k}+\varepsilon_{24}^{k}\right) \mathbf{j}+\left(\varepsilon_{14}^{k}-\varepsilon_{23}^{k}\right) \mathbf{k}\right\} .
$$

From (5.2) here we have $M_{\mu}=\frac{1}{2\left(1+4 \mu^{2}\right)\left(1+\mu+2 \mu^{2}\right)}$. Since $k_{i}=\mu$, in (5.5) we can write $\varepsilon_{i j}^{\mu}$ instead of $\varepsilon_{i j}^{k}$.

If we consider the 0 -form

$$
\omega=\sum_{\mu} M_{\mu}(1-\mu) x^{\mu}, \quad \mu \in \mathbb{Z}
$$

and use the following relation (see the proof of Proposition 4.5)

$$
\bar{e} \cup e=2\left\{\left(\varepsilon_{12}-\varepsilon_{34}\right) \mathbf{i}+\left(\varepsilon_{13}+\varepsilon_{24}\right) \mathbf{j}+\left(\varepsilon_{14}-\varepsilon_{23}\right) \mathbf{k}\right\},
$$

then $F$ can be written as

$$
F=\omega \cup \bar{e} \cup e .
$$

In view of Corollary $4.6 F$ is anti-self-dual, i.e., $F=-\tilde{\imath} * F$. Thus under condition (5.4) $A$ with components (5.1) describes an anti-instanton.

In the same manner we can see that the following quaternionic 1-form

$$
A=\operatorname{Im}(f \cup \bar{e}),
$$

where $f$ has the components

$$
f_{k}=\frac{\kappa}{1+|\kappa|^{2}}
$$

leads to an instanton solution of (4.24). Indeed, substituting (5.8) and (5.9) in (4.16) we now obtain

$$
\begin{aligned}
F_{k}^{12} & =\left\{-M_{1}\left(1+k_{2}^{2}-k_{1}^{2}-k_{1}\right)-M_{2}\left(1+k_{1}^{2}-k_{2}^{2}-k_{2}\right)\right\} \mathbf{i} \\
& +\left\{M_{1}\left(k_{4} k_{1}-k_{2} k_{3}\right)+M_{2}\left(k_{3} k_{2}-k_{4} k_{1}\right)\right\} \mathbf{j} \\
& +\left\{M_{1}\left(-k_{2} k_{4}-k_{1} k_{3}\right)+M_{2}\left(k_{1} k_{3}+k_{2} k_{4}\right)\right\} \mathbf{k} \\
& +M_{1}\left(k_{1} k_{2}+k_{2}\right)-M_{2}\left(k_{1} k_{2}+k_{1}\right) \\
F_{k}^{13} & =\left\{M_{1}\left(-k_{2} k_{3}-k_{1} k_{4}\right)+M_{3}\left(k_{1} k_{4}+k_{2} k_{3}\right)\right\} \mathbf{i} \\
& -\left\{M_{1}\left(1+k_{3}^{2}-k_{1}^{2}-k_{1}\right)+M_{3}\left(1+k_{1}^{2}-k_{3}^{2}-k_{3}\right)\right\} \mathbf{j} \\
& +\left\{M_{1}\left(k_{1} k_{2}-k_{3} k_{4}\right)+M_{3}\left(k_{3} k_{4}-k_{1} k_{2}\right)\right\} \mathbf{k}
\end{aligned}
$$




$$
\begin{aligned}
& +M_{1}\left(k_{1} k_{3}+k_{3}\right)-M_{3}\left(k_{1} k_{3}+k_{1}\right), \\
F_{k}^{14} & =\left\{M_{1}\left(k_{1} k_{3}-k_{2} k_{4}\right)+M_{4}\left(k_{2} k_{4}-k_{1} k_{3}\right)\right\} \mathbf{i} \\
& +\left\{M_{1}\left(-k_{3} k_{4}-k_{1} k_{2}\right)+M_{4}\left(k_{1} k_{2}+k_{3} k_{4}\right)\right\} \mathbf{j} \\
& -\left\{M_{1}\left(1+k_{4}^{2}-k_{1}^{2}-k_{1}\right)+M_{4}\left(1+k_{1}^{2}-k_{4}^{2}-k_{4}\right)\right\} \mathbf{k} \\
& +M_{1}\left(k_{1} k_{4}+k_{4}\right)-M_{4}\left(k_{1} k_{4}+k_{1}\right), \\
F_{k}^{23} & =\left\{M_{2}\left(-k_{2} k_{4}+k_{1} k_{3}\right)+M_{3}\left(-k_{1} k_{3}+k_{2} k_{4}\right)\right\} \mathbf{i} \\
& +\left\{M_{2}\left(k_{3} k_{4}+k_{1} k_{2}\right)-M_{3}\left(k_{1} k_{2}+k_{3} k_{4}\right)\right\} \mathbf{j} \\
& -\left\{M_{2}\left(1+k_{3}^{2}-k_{2}^{2}-k_{2}\right)+M_{3}\left(1+k_{2}^{2}-k_{3}^{2}-k_{3}\right)\right\} \mathbf{k} \\
& +M_{2}\left(k_{2} k_{3}+k_{3}\right)-M_{3}\left(k_{2} k_{3}+k_{2}\right), \\
F_{k}^{24} & =\left\{M_{2}\left(k_{2} k_{3}+k_{4} k_{1}\right)-M_{4}\left(k_{1} k_{4}+k_{2} k_{3}\right)\right\} \mathbf{i} \\
& +\left\{M_{2}\left(1+k_{4}^{2}-k_{2}^{2}-k_{2}\right)+M_{4}\left(1+k_{2}^{2}-k_{4}^{2}-k_{4}\right)\right\} \mathbf{j} \\
& +\left\{M_{2}\left(k_{1} k_{2}-k_{3} k_{4}\right)+M_{4}\left(k_{3} k_{4}-k_{1} k_{2}\right)\right\} \mathbf{k} \\
& +M_{2}\left(k_{2} k_{4}+k_{4}\right)-M_{4}\left(k_{2} k_{4}+k_{2}\right), \\
& \\
F_{k}^{34}= & -\left\{M_{3}\left(1+k_{4}^{2}-k_{3}^{2}-k_{3}\right)+M_{4}\left(1+k_{3}^{2}-k_{4}^{2}-k_{4}\right)\right\} \mathbf{i} \\
& +\left\{M_{3}\left(-k_{2} k_{3}+k_{1} k_{4}\right)+M_{4}\left(-k_{1} k_{4}+k_{2} k_{3}\right)\right\} \mathbf{j} \\
& +\left\{M_{3}\left(k_{2} k_{4}+k_{1} k_{3}\right)-M_{4}\left(k_{1} k_{3}+k_{2} k_{4}\right)\right\} \mathbf{k} \\
+ & M_{3}\left(k_{3} k_{4}+k_{4}\right)-M_{4}\left(k_{3} k_{4}+k_{3}\right) . \\
&
\end{aligned}
$$

Again, under condition (5.4) we can write $F$ as

$$
F=\sum_{\mu} M_{\mu}(2 \mu-2)\left\{\left(\varepsilon_{12}^{\mu}+\varepsilon_{34}^{\mu}\right) \mathbf{i}+\left(\varepsilon_{13}^{\mu}-\varepsilon_{24}^{\mu}\right) \mathbf{j}+\left(\varepsilon_{14}^{\mu}+\varepsilon_{23}^{\mu}\right) \mathbf{k}\right\},
$$

where $\mu \in \mathbb{Z}$. Therefore

$$
F=\omega \cup e \cup \bar{e},
$$

where $\omega$ is given by (5.6). Thus the discrete curvature form (5.10) is self-dual and we can say that (5.8) describes an instanton.

Now to complete the analogy with the continual case we describe more precisely how the anti-instanton given by (5.1) behaves as $|\kappa| \rightarrow \infty$. It is clear that $f_{k}$ is asymptotically $\frac{\bar{k}}{|\kappa|^{2}}=\kappa^{-1}$. Then

$$
A \sim \operatorname{Im}\left(x^{-1} \cup d^{c} x\right) \text { as }|\kappa| \rightarrow \infty .
$$

Here $x$ is given by (4.10). By virtue of Proposition 4.3 the discrete curvature $F=0$ at infinity. 
Proposition 5.2. The anti-instanton (5.1) has the same form at $\infty$ as it has near 0.

Proof. Introduce the quaternionic 0-form

$$
y=\sum_{k} y_{k} x^{k}, \quad \text { where } \quad y_{k}=\frac{1}{\kappa}
$$

and remind $\kappa=k_{1}+k_{2} \mathbf{i}+k_{3} \mathbf{j}+k_{4} \mathbf{k}$. Clearly, $y=x^{-1}$. We first compute $x \cup f \cup e \cup x^{-1}$, where $f$ is given by (5.1). To do this, take (4.10), (4.11) and use the $\cup$-product definition. We have

$$
\begin{aligned}
x \cup f \cup e & =\left(\sum_{k} \kappa x^{k}\right) \cup\left(\sum_{k} \frac{\bar{\kappa}}{1+|\kappa|^{2}} x^{k}\right) \cup e \\
& =\left(\sum_{k} \frac{|\kappa|^{2}}{1+|\kappa|^{2}} x^{k}\right) \cup e=e-\left(\sum_{k} \frac{1}{1+|\kappa|^{2}} x^{k}\right) \cup e \\
& =d^{c} x-\left(\sum_{k} \frac{1}{1+|\kappa|^{2}} x^{k}\right) \cup d^{c} x .
\end{aligned}
$$

From this by (4.5) we get

$$
x \cup f \cup e \cup x^{-1}=-x \cup d^{c} x^{-1}+\left(\sum_{k} \frac{\kappa}{1+|\kappa|^{2}} x^{k}\right) \cup d^{c} x^{-1} .
$$

Now gauge transform the form $f \cup e$ by the gauge transformation $g=x^{-1}$. We must take the imaginary part of (4.13). This yields by (5.12)

$$
\begin{aligned}
\operatorname{Im}\left(g^{-1} \cup f \cup e \cup g+g^{-1} \cup d^{c} g\right) & =\operatorname{Im}\left(\left(\sum_{k} \frac{\kappa}{1+|\kappa|^{2}} x^{k}\right) \cup d^{c} x^{-1}\right) \\
& =\operatorname{Im}\left(\left(\sum_{k} \frac{\bar{y}_{k}}{1+\left|y_{k}\right|^{2}} x^{k}\right) \cup d^{c} y\right) .
\end{aligned}
$$

Hence the gauge transformed anti-instanton $A$ has precisely the form (5.11) near $y=0$.

The same conclusion can be drawn for the instanton (5.8).

In the continual theory Proposition 5.2 shows that the anti-instanton (or instanton) extends to the 4-sphere $S^{4}$. This follows from the fact that $S^{4}$ can be obtained from $\mathbb{R}^{4}$ by adding the point at infinity, i.e., $S^{4} \simeq \mathbb{R}^{4} \cup\{\infty\}$. To obtain the same result for our discrete model we need to construct a suitable combinatorial analog of the 4-sphere. It would be interesting to connect the above constructions with discrete model of $S^{4}$ described in [21]. This connection must be investigated and we hope to treat its further in future work.

\section{References}

[1] АтіYah, M.F., Geometry of Yang-Mills Fields, Lezione Fermiane, Scuola Normale Superiore Pisa, 1979. 
[2] De Beaucé, V., Sen, S. And Sexton, J.C., Chiral dirac fermions on the lattice using geometric discretisation, Nucl. Phys. B (Proc. Suppl.), 129-130 (2004), 468-470.

[3] Belavin, A., Polyakov, A., Schwartz, A. And Tyupkin, Y., Pseudoparticle solutions of the Yang-Mills equations, Phys. Lett. B, 59 (1976), 86-87.

[4] Castellani, L. And Pagani, C., Finite Group Discretization of Yang-Mills and Einstein Actions, Annals of Physics, 297 (2002), no. 2, 295-314.

[5] Corrigan, E. AND FAirlie, D.B., Scalar field theory and exact solutions to a classical $S U(2)$-gauge theory, Phys. Lett. B, 67 (1977), 69-71.

[6] Dezin, A.A., Multidimensional Analysis and Discrete Models, CRC Press, Boca Raton, 1995.

[7] Dezin, A.A., Models generated by the Yang-Mills equations, Differentsial'nye Uravneniya, 29 (1993), no. 5, 846-851; English translation in Differential Equations, 29 (1993), no. 5, 724-728.

[8] Freed, D. and Uhlenbeck, K., Instantons and Four-Manifolds, Springer-Verlag, 1984.

[9] DE Forcrand, Ph. AND Jahn, O., Comparison of $S O(3)$ and $S U(2)$ lattice gauge theory, Nuclear Physics B, 651 (2003), 125-142.

[10] Gonzalez-Arroyo, A. And Montero, A., Self-dual vortex-like configurations in $S U(2)$ Yang-Mills theory, Physics Letters B, 442 (1998), 273-278.

[11] JACKiw, R., NoHL, C. AND REBBi, C., Conformal properties of pseudo-particle configurations, Phys. Rev., 150 (1977), 1642-1646.

[12] Kamata, M. And Nakamula, A., One-parameter family of selfdual solutions in classical Yang-Mills theory, Physics Letters B, 463 (1999), 257-262.

[13] Nakamula, A., Selfdual solution of classical Yang-Mills fields through a $q$-analog of ADHM construction, Reports On Mathematical Physics, 48 (2001), 195-202.

[14] NaSh, C. AND Sen, S., Toplogy and Geometry for Physicists, Acad. Press, London, 1989.

[15] Nishimura, J., Four-dimensional $N=1$ supersymmetric Yang-Mills theory on the lattice without fine-tuning, Phys. Lett.B, 406 (1997), no. 3, 215-218.

[16] Seiler, E., Gauge theories as a problem of constructive quantum field theory and statistical mechanics, Lecture Notes in Physics 159, Springer-Verlag, 1982. 
[17] Sen, S., Sen, S., Sexton, J.C. And Adams, D., A geometric discretisation scheme applied to the Abelian Chern-Simons theory, Phys. Rev. E, 61 (2000), 3174-3185; arxiv: hep-th/0001030.

[18] Shabanov, S., Infrared Yang-Mills theory as a spin system. A lattice approach, Phys. Lett.B, 522 (2001), no. 1-2, 201-209.

[19] Sushch, V., Gauge-invariant discrete models of Yang-Mills equations, Mat. Zametki, 61 (1997), no. 5, 742-754; English translation in Mathematical Notes, 61 (1997), no. 5, 621-631.

[20] Sushch, V., Discrete model of Yang-Mills equations in Minkowski space, Cubo A Math. Journal, 6 (2004), no. 2, 35-50.

[21] Sushch, V., A gauge-invariant discrete analog of the Yang-Mills equations on a double complex, Cubo A Math. Journal, 8, (2006), no. 3, 61-78. 